\title{
Gobernaciones y Nueva Gestión Pública en Venezuela
}

\section{Introducción}

Desde los años ochenta, se producen cambios en la administración pública, especialmente en los países desarrollados, dando lugar a un estilo de conducción del aparato público denominado Nueva Gestión Pública, sobre el cual ha habido una gran producción intelectual, especialmente de tipo propositiva, que en su mayoría le asigna a dicho modelo, objetivos de democracia y eficiencia. Este conocimiento ha constituido un aspecto subjetivo favorable al avance de una nueva gestión pública en los distintos países donde se realizan acciones para torno a la modernización del aparato público, especialmente después del fracaso de medidas cuyo centro de atención estuvo en la reducción del tamaño del Estado, denominadas reformas de primera generación.

En Venezuela asistimos desde comienzos de los años noventa a un avance silencioso de ese paradigma en la gestión pública. Pasamos de modo selectivo, a diferencia de las propuesta de reforma integral de la administración pública de los años sesenta, setenta y comienzos de los ochenta, de una administración pública denominada burocrático populista (Ochoa, et al, 1996), muy distante de las prescripciones legales weberianas, a una práctica administrativa con muchos elementos del paradigma denominado nueva gestión pública, aunque alejada aún de las propuestas cuyos propósitos formales son la eficiencia y la democracia.

También asistimos desde fines de los ochenta a una reforma del Estado. Uno de los ejes lo constituye la descentralización político territorial hacia el nivel estadal, con lo cual las gobernaciones, instancias de dirección de este nivel, asumen importantes funciones del Estado y a la vez se les

Haydée Ochoa Henríquez es doctora en Estudios del Desarrollo, investigadora del Centro de Estudios de la Empresa de la Facultad de

Ciencias

Económicas y

Sociales de la

Universidad del

Zulia

(Venezuela) y directora de la Revista

Venezoelana de Gerencia.

Contato: haydeeochoa@ iamnet.com 
presiona por cambios en su gestión, hecho que en algunos casos forma parte de los convenios de transferencia de competencias. Producto de esto las gobernaciones desde los mismos inicios de la descentralización han llevado adelante proyectos de reforma, para lo cual han movilizado grandes recursos financieros y humanos.

Lo expuesto nos anima a explorar la presencia del paradigma de La Nueva Gestión Pública en la reforma administrativa llevada adelante por las gobernaciones venezolanas desde los inicios de la descentralización. Manejamos el supuesto que se han venido incorporando propuestas dirigidas a reducir el aparato estatal y a incrementar su eficiencia, dejando de lado, aquéllas con propósitos de profundización de la democracia para viabilizar una mayor justicia social.

La metodología utilizada consistió en contrastar las principales tendencias y propuestas sobre una nueva gestión pública, con los cambios adelantados por algunas gobernaciones venezolanas para lo cual se analizó información contenida en: documentos oficiales de varias gobernaciones, proyectos e informes de los organismos multilaterales financistas de la reforma, otros documentos con información empírica y en entrevistas semiestructuradas realizadas a líderes del proceso, así como a otros actores involucrados.

\section{¿Qué es la nueva gestión pública? Una precisión preliminar}

El término nueva gerencia pública, o nueva gestión pública o gerencia pública sirve para designar un nuevo modelo de administración pública, en palabras de Barzelay (2001:11): "La Nueva Gerencia Pública (NGP) se origina como un dispositivo conceptual inventado con el propósito de estructurar la discusión académica sobre los cambios contemporáneos en la organización y el gerenciamiento de la rama ejecutiva del gobierno". Según este autor, la referencia de mayor tiempo en el uso del término, data de 1991, sin embargo para dar cuenta de una nueva gestión pública, en América Latina se utilizó desde mucho antes, el término Gerencia Pública (KLIKSBERG, 1989), cuando para identificar al viejo modelo, se utilizaba el concepto "Administración Pública".

El nuevo modelo identificado bajo diversos términos y nociones, algunos autores lo oponen al paradigma ideal weberiano el cual debió formalmente orientar la administración pública, debido a su consideración en el marco institucional por distintos países, sin embargo en la práctica el paradigma de la Nueva Gestión Pública sustituye un estilo de dirección pública alejado de las propuestas weberianas, vinculado a los requerimientos 
del Estado Keynesiano, mejor conocido como Estado de bienestar, cuya

versión latinoamericana es el Estado Populista.

Las características más resaltantes de la nueva gestión pública hoy en día en muchos países, pero más claramente en los países pioneros, tales como Nueva Zelandia, Reino Unido y Australia, apuntan, aunque con diferencia en grados y pasando por varias etapas en cada caso por la vía de ensayo y error, a: 1) La separación organizativa del nivel de definición de políticas, de su implementación y una mayor capacidad de actuación de éste, a través de la creación de unidades descentralizadas administrativamente, 2) La evaluación y control por resultados con base en objetivos y no sobre insumos y procesos, en Nueva Zelandia además de los resultados se considera el impacto en la sociedad 3) Desmantelamiento del servicio civil, especialmente de los niveles gerenciales, en los cuales se ha introducido la contratación por tiempos cortos, cuya renovación está sujeta al rendimiento y 4) Sistemas de información contable y su publicación (Arellano et al, 2001; Barzelay, 1998).

Algunas de estas tendencias son recogidas en propuestas latinoamericanas, es el caso de la creación de agencia descentralizadas administrativamente y el control por resultados, pero otros aspectos constituyen elementos clave en América Latina, son ellos: la descentralización hacia niveles subnacionales, la transferencia de servicios a organizaciones de la sociedad civil y la profesionalización de la alta burocracia (CLAD; 1998), esto último en contradicción con la tendencia flexibilizadora.

Nuestra tesis es que estos cambios descritos por lo general asépticamente, en palabras de Omar Guerrero (1999:11), "sin grandes aspiraciones académicas y científicas", son producto de la necesidad de la globalización y la economía de mercado expandida, de un Estado eficiente, es decir, de responder a los cambios institucionales asignados para la promoción de la globalización y la economía de mercado, al menor costo posible en materia laboral y social, rubros incrementados por el Estado de bienestar, los cuales resultaban funcionales a economías restringidas a la producción para el consumo interno. Esto explica que el centro de atención sea la reducción de los costos laborales y la eliminación de la gratuidad de los servicios.

\section{Gobernaciones y Nueva Gestión Pública en la Reforma del Estado en Venezuela}

En el marco de una profunda crisis del país en todos los órdenes y de presiones de los sectores dominantes para que el Estado promoviera los cambios sobre los cuales avanzaba el resto de América Latina, desde 
mediados de los ochenta se realizan intentos de reforma del Estado en Venezuela dirigidos a superar los históricos esfuerzos centrados solo en los cambios de la administración pública. Sin embargo es a fines de los ochenta, cuando en el contexto del inicio de un nuevo gobierno, el Estado venezolano promueve la reestructuración económica acompañada de la descentralización político territorial, cuyo proyecto venía gestándose desde mediados de la década, el cual fue necesario apresurar como estrategia de búsqueda de legitimación a raíz de grandes protestas en contra de la reestructuración económica de corte neoliberal.

La descentralización político territorial previó por una parte, el traspaso de competencias del gobierno central al gobierno estadal, instancia intermedia entre el nivel nacional y el municipal, de esta manera las gobernaciones, organizaciones responsables de la dirección estadal, pasan a tomar importancia significativa al asumir competencias de tipo económico que nunca habían tenido y amplían notablemente sus responsabilidades de tipo social. Por otra parte, se previó la elección directa de los gobernadores, quienes hasta entonces eran nombrados por el Presidente de la República de las filas del partido que lo llevó al poder. Estos políticas pasaron por la aprobación de la ley Orgánica de Descentralización, Delimitación y Transferencias de Poder Público y la Ley de Elección y Remoción de Gobernadores y Alcaldes.

En este proceso las gobernaciones asumen competencias en condiciones de exclusividad, en materia de puertos, aeropuertos, explotación de salinas, puentes sobre ríos, el puente sobre el lago de Maracaibo y vialidad entre otras de menor importancia, con ello pasan a tener un rol significativo en la buena marcha de la economía estadal, nacional y hasta internacional en razón de la vinculación de algunas de estas actividades con el comercio internacional y a manejar cuantiosos recursos provenientes de la prestación de estos servicios, los cuales siempre han tenido un carácter empresarial.

Compartidas con otros niveles estatales, las gobernaciones, reciben también del gobierno central competencias en materia social, aunque a través de una forma más lenta y compleja en relación al traslado de las competencias exclusivas. De particular relevancia en este sentido, es el traspaso de los servicios de salud, los cuales eran responsabilidad de la administración pública nacional. Esto da cuenta del nuevo papel de las gobernaciones en la reforma del Estado, impulsada conjuntamente con la reestructuración económica.

Acompañó a la descentralización político-territorial, la creación del Fondo Intergubernamental para la Descentralización (FIDES), creado para apoyar financieramente el proceso y la Ley de Asignaciones Económicas Especiales, la cual beneficia a los estados mineros y petroleros, con ello se 
incrementa la capacidad financiera de las gobernaciones y en consecuencia

su posición política frente a los otros niveles estatales y a la sociedad.

En este contexto los gobernadores de estados, son actores políticos importantes en el país y muchos de ellos se programan para competir en elecciones presidenciales, pasando esto por la reelección en su cargo, incidiendo en la búsqueda de una gestión eficiente y así poder demostrar condiciones para sus aspiraciones políticas de mayor envergadura, alimentándose esto además, de la relevancia asignada a la capacidad gerencial de los gobernantes hoy en día.

Paralelo a la descentralización político-territorial, se avanza silenciosamente y por la vía selectiva en un proceso de reforma de algunos espacios de la administración pública. Así a través de la estrategia selectiva y sin mucho ruido, al menos mientras se implanta la reforma, la vieja administración pública, conocida como burocrático populista, en poca medida vinculada al paradigma weberiano, se sustituye con el modelo de la Nueva Gestión Pública, dejando atrás los intentos frustrados de reforma integral expresados en varios proyectos que pretendían por la vía de decreto cambiar toda la administración del aparato público.

Algunos proyectos elaborados desde la Comisión Presidencial para la Reforma del Estado (COPRE) que se plantearon una reforma a nivel de toda la administración pública, tal es el de la Profesionalización de la Gerencia Pública, no trascendieron el nivel formal, además de la estrategia, le restó viabilidad al proyecto, el hecho de ir contra la tendencia flexibilizadora del servicio civil.

La reforma más visible inicialmente a nivel de logros, es la del aparato tributario, la cual fue impulsada desde los primeros años de la década de los noventa, con un papel activo de los organismos multilaterales (FMI, BM, BID y CIAT), vinculada a una nueva política tributaria de notoria relación con la nueva orientación económica. Si bien esta reforma aplicó el modelo promovido por los organismos internacionales en toda América Latina, es útil hacer referencia a sus características, las cuales siguen las tendencias de las reformas de la administración pública en otros países y sectores. Entre las características más resaltantes de la reforma tributaria se encuentran: Cambios en la estructura organizativa para delimitar claramente el nivel de elaboración de políticas del de ejecución y darle a éste mayor autonomía con base en el control por resultados, la aplicación de principios meritocráticos en la selección y el ascenso del personal, la simplificación de los procesos, el traspaso de algunas funciones a la banca y empresas privadas y nuevas relaciones con los contribuyentes con un papel fundamental de estrategias modernas de marketing (Ochoa y Gamboa, 1999).

A este proceso de reforma se sumaron la mayoría de las gobernaciones del país, desde el mismo momento que se produjo la 
descentralización político territorial, promovido esto por el Gobierno Nacional desde varias instancias, entre ellas: La Oficina Central de Coordinación y Planificación (COORDIPLAN), hoy Ministerio de Planificación; el Fondo de Inversión Social (FONVIS) y el Fondo Intergubernamental para la Descentralización (FIDES), instituciones éstas que disponían de recursos financieros y apoyo técnico de los organismos multilaterales (BID, Banco Mundial y PNUD), dirigidos a apoyar la descentralización y el fortalecimiento institucional (Fuenmayor y Ochoa, 2001).

La dinámica por la cual ha pasado la reforma de las gobernaciones hacia una Nueva Gestión Pública, ha sido muy diferente en relación a otros aparatos, como veremos a continuación.

\section{La reducción del tamaño del aparato estadal y la conformación de redes con organizaciones no estatales.}

La privatización a través de la venta de acciones y de activos del Estado fue una política que caracterizó los inicios de la reforma del Estado en muchos países y que condujo a la reducción considerable de la administración pública, de ello no escapó nuestro país. Pero paralelamente se utilizado otras estrategias para reducir el aparato estatal, que han sido menos visibles y en las cuales se insertaron las gobernaciones desde que se inició el proceso de descentralización político-territorial, lo que de hecho significa un avance en términos de asumir una Nueva Gestión Pública.

\section{Concesiones al sector privado para las competencias vinculadas a la economía}

La creación de infraestructura adecuada para la economía, en especial de transporte, es sin lugar a dudas una cuestión fundamental en una economía de mercado, por ello ocupó un espacio importante en el Foro Empresarial de las Américas realizado en 1996, en el cual quedó expuesto que "eliminar progresivamente las barreras al comercio y a la inversión en el hemisferio para el 2005, requiere tratamiento de los temas relacionados con los distintos modos de transporte y con la infraestructura vial, ferroviaria, portuaria y aeroportuaria" (Boulton et al, 1996). Entre las recomendaciones que se hacen en el mencionado evento está "Crear las condiciones para la participación organizada del sector privado en la implementación de la infraestructura física y en la definición e instrumentación de las políticas y proyectos destinados a este objetivo" (Boulton et al, 1996). 
En Venezuela, con el proceso de descentralización, se trasladaron en condiciones de exclusividad a las Gobernaciones, los servicios de infraestructura a la economía, concretamente los puertos, vialidad, aeropuertos y el Puente sobre el Lago de Maracaibo, los cuales en su mayoría fueron entregados en concesión al sector privado a través de diversas estrategias administrativas, con algunas diferencias especialmente al comienzo, pero con tendencia finalmente a la creación de instituciones con cierto grado de autonomía responsables de la ejecución de las políticas y del control de las concesiones.

Toda la infraestructura portuaria estatal de uso comercial, fue descentralizada ${ }^{1}$ y su operación entregada en concesión al sector privado ${ }^{2}$, en un proceso simultáneo de descentralización y concesión, lo que es producto de condiciones establecidas por el gobierno central para el traspaso de estos servicios a las gobernaciones. El servicio más comprometido con la privatización en el proceso de descentralización fue el de Puertos, por donde circula aproximadamente el 97\% del intercambio comercial de mercancías con otros países. La privatización del servicio portuario "fue decidida antes de producirse la descentralización, lo cual fue aceptado por los gobernadores. Por ejemplo los planes de privatización de los puertos, fueron ampliamente difundidos cuando se reestructuraba el Instituto Nacional de Puertos (INP) para su liquidación" (Ochoa, 2001: 109).

En cuanto a los aeropuertos ${ }^{3}$, el proceso de privatización del servicio, no tuvo la misma celeridad que los puertos, pero se produjo en todo el país, pasando desapercibido. La privatización de servicios básicos y de apoyo en los aeropuertos pasó por el establecimiento de tarifas por el uso de los aeropuertos, a los pasajeros, esto ha permitido realizar cuantiosas inversiones para modernizar el servicio cuya demanda se ha intensificado. Para los sectores económicos, en los actuales momentos es una necesidad "Modernizar, en el corto plazo, los aeropuertos de tráfico internacional, tanto física como tecnológicamente para adecuarlos a los parámetros de seguridad establecidos en los manuales y disposiciones de la OACI" (Boulton et al; 1996).

Finalmente entre las competencias descentralizadas vinculadas a la economía y a la dotación de infraestructura de transporte se encuentra la vialidad, Históricamente había sido un servicio gratuito, condición modificada con la privatización, generando cuantiosos ingresos permitiendo mejorar el servicio. Sobre la infraestructura vial también se pronunció el Foro Empresarial de las Américas en 1996, el cual señala al respecto lo siguiente: "se requiere de la puesta en vigencia en todo el ámbito continental, del 'Sistema de concesiones'. Esto estimulará y facilitará que la inversión privada fluya con confianza económica y seguridad jurídica hacia el sector de infraestructura física, principalmente en lo que corresponde a vialidad" (Boulton et al; 1996). 
Con la privatización de los servicios de infraestructura a la economía, ha sido reducido a su mínima expresión el número de trabajadores de las gobernaciones dedicados a la operación y gestión de los servicios en referencia, a modo de ejemplo, en Puerto Cabello se pasó de 5000 empleados públicos antes de la descentralización a 151 dependientes del Instituto Puerto Autónomo de Puerto Cabello, creado por la Gobernación (Mascareño, et al, 2000). Para algunos autores la razón de esta privatización está vinculada a la búsqueda de eficiencia requerida en los puertos para el buen funcionamiento del mercado internacional, sin embargo teniendo en consideración que la capacidad para lograr eficiencia no es exclusiva del sector privado, que ésta también puede ser lograda por el Estado, las razones a nuestro juicio están vinculadas a la necesidad de lograr eficiencia con la reducción de costos y conflictos laborales, más garantizada con el sector privado, dadas las mayores posibilidades de flexibilización laboral de ese sector, esto además ha tenido lugar en el contexto de presiones por parte del sector privado, como hemos visto, para asumir la gestión de los servicios de infraestructura de transporte.

El traspaso de funciones del Estado por la vía de concesión al sector privado modifica profundamente la administración pública, incide en la conformación de una Nueva Gestión Pública. En primer lugar, en las relaciones entre los ciudadanos y el Estado, en la prestación de servicios por parte de éste, media el sector privado ${ }^{4}$, el cual tiene propósitos de rentabilidad de su capital, muy diferentes a los del Estado, cuestión que le imprime carácter empresarial a las relaciones, en las cuales el ciudadano es un cliente. En segundo lugar, el número de funcionarios del Estado se reduce y se transforman las condiciones de trabajo, cuyo rasgo principal es la flexibilización que se facilita con la pertenencia a entes autónomos y semiautónomos.

\section{Promoción de ONGs para las funciones sociales de las gobernaciones}

La reducción del aparato estatal se ha dado también a través del traspaso de funciones sociales del Estado a distintas organizaciones no gubernamentales, del tercero y cuarto sector, esto a nuestro juicio está vinculada a la necesidad de reducir el gasto social. El traspaso de funciones a las ONGs no ha tenido lugar en las gobernaciones con la misma celeridad que la privatización, explicable por la desconfianza del Estado respecto a la aplicación de criterios de eficiencia por parte de las ONGs, sin embargo poco a poco las gobernaciones promueven la creación de estas organizaciones para darles la responsabilidad de algunas funciones sociales asumidas con el proceso de descentralización político-territorial, lo cual encuentra condiciones favorables en el desempleo, en la búsqueda de 
satisfacción de necesidades no cubiertas y en objetivos de control social por parte de algunos grupos políticos.

Según Mascareño et al (2000: 128), en las entidades federales, han surgido organizaciones de la sociedad civil "al ritmo de la descentralización y se han especializado sectorialmente en educación, salud, vivienda, cultura, deporte o programas sociales", las cuales según los autores "se han convertido en facilitadoras de la gestión gubernamental", "han generado un efecto demostración sobre las posibilidades y formas de ejecutar servicios sin administración gubernamental" y entre otros beneficios le atribuyen contribución al mejoramiento de los servicios.

$\mathrm{Si}$ bien las ONGs se promueven en todos los sectores de tipo social, es en el campo de la salud, vivienda, Deporte y Desarrollo Social donde se observa una acción programada por parte de algunas gobernaciones para transferir funciones a organizaciones de la sociedad civil, aunque es en salud donde se observa una tendencia a sustituir la gestión directa por la gestión de $\mathrm{ONGs}^{5}$, a pesar de estar formalmente previstas las Juntas Sociosanitarias a través de las cuales debe darse una participación de la sociedad civil en los servicios de salud, modelo que tiende a aplicarse en el Estado Carabobo.

En cuanto a la promoción de ONGs en materia de salud, el caso emblemático es el de la Gobernación del Estado Lara, donde "más de 350 organizaciones civiles participan en la gestión de la salud en programas que van desde el manejo de instituciones, el saneamiento ambiental, la administración de la red ambulatoria, de la red de ambulancias hasta la administración de farmacias sociales, de programas de la $3^{\text {a }}$ edad y de programas de prevención entre otros... más de 70 ambulatorios que tienen bajo su área de influencia a más de $75 \%$ de la población estadal, se encuentran bajo cogestión con la sociedad civil" (Mascareño et al, 2000: 136). Estas organizaciones de la sociedad civil en materia de salud se vinculan a más de quince programas de la gobernación del Estado Lara en la materia.

En el Estado Zulia, a partir de un caso considerado exitoso, se programa en los actuales momentos el traspaso de gran parte de las unidades de salud a organizaciones de la sociedad civil. La experiencia en referencia consistió en la entrega de un ambulatorio a la Fundación Venezolana de Medicina Familiar (FUNVEMEFA), organización corporativa creada por médicos de Medicina Familiar, la cual a través del lema de Combatir el PGS, donde P: es Clientelismo Político, G: Gremialismo y S: Sindicatos (Gruber, citado por Ochoa y Rodríguez, 2002), ha promovido la gestión de la salud desde Fundaciones y con la contratación de personal a través de organizaciones llamadas microempresas, sustituyendo la relación laboral con una relación mercantil. El Dr. Gruber, líder de este modelo, fue 
nombrado Secretario de Salud en la Gobernación del Zulia desde donde realizó un trabajo tendiente a difundir el modelo en todos los centros de salud de la gobernación.

La experiencia de incorporación de la Sociedad civil en la gestión de la salud, ha pasado por el cobro del servicio, el cual históricamente ha sido prestado por el Estado de forma gratuita, esto está vinculado a que el traslado del servicio a la sociedad civil se ha dado paralelo a una reducción de la asignación de recursos, en consecuencia la principal fuente financiera la constituye el cobro del servicio, de esta forma paulatinamente el Estado se desprende de su responsabilidad social en materia de salud, minimizando al mismo tiempo, la administración pública en la materia, al ser sustituidos los funcionarios con "microempresarios".

El traspaso de funciones del Estado a la sociedad civil, modifica el modelo de gestión pública. En las relaciones del Estado con los ciudadanos median organizaciones, aparentemente con funciones de bienestar colectivo, pero en su lucha por la obtención de los recursos del Estado, terminan lesionando los intereses sociales. Según Petras (1999):" La ayuda de ONG's afecta a sectores pequeños de la población al generar entre comunidades competencia por recursos escasos, que generan distinciones insidiosas y rivalidades inter e intra comunitarias, socavando así la solidaridad de clase. Lo mismo es válido entre profesionales: cada quien crea su ONG para solicitar fondos del exterior. Compiten al presentar propuestas más convenientes para los donadores ultramarinos, al tiempo que afirman hablar en nombre de sus seguidores".

\section{Las microempresas sustituyen a los funcionarios}

Las microempresas constituyen hoy en día una estrategia promovida por el Estado buscando dar respuesta a las altas tasas de desempleo, dando lugar a instituciones y fondos para ayuda financiera por parte del Estado en sus distintos niveles. Pero además es una estrategia para reducir las relaciones laborales, las cuales se convierten en relaciones mercantiles, fenómeno iniciado en nuestro país en el sector privado hace mucho tiempo, pero promovido intensamente en los últimos años y viene siendo utilizado también por la administración pública.

La promoción de microempresarios para reducir el personal del Estado, escasamente ha sido estudiada, explicable por su reciente uso, expondremos aquí el caso de la Gobernación del Estado Zulia. La experiencia se inició con la conversión del personal médico, paramédico y administrativo en microempresarios por parte de FUNVEMEFA, reduciendo así el costo y los conflictos laborales, estrategia, programada para extenderla a todo el sistema de salud. Pero adicionalmente como 
parte de un plan de reconversión laboral "se le ofreció a los obreros la posibilidad de acogerse a un programa que contemplaba tanto la capacitación como el apoyo técnico y financiero para que conformaran microempresas, que luego serían contratistas de la Gobernación para la prestación de servicios de limpieza, mantenimiento, mensajería, reproducción, entre otros" (Obregón y Carucci, 2000: 64). Esta estrategia se llevó a cabo en la Secretaría de Administración y sus resultados son considerados exitosos, después de crearse 40 microempresas con extrabajadores de la mencionada Secretaría, por ello, de acuerdo a Obregón y Carucci (2000:64) "se decidió aplicar un plan similar en el resto del aparato administrativo, sin embargo, el proceso electoral del 2000 relegó el tema del ajuste y la reconversión en la agenda de los directivos de la Gobernación y provocó una virtual paralización del proceso". Por la vía selectiva se avanza en la aplicación de esta estrategia en las Secretarías de Obras Públicas, Educación y la de Cultura además del Despacho del Gobernador.

Recientemente (2002) el Dr. Félix Gruber, iniciador del uso de la estrategia de microempresas para sustituir al personal del Estado en el área de salud, fue nombrado Director de la Fundación para la Modernización de la Administración Pública, de la Gobernación del Estado Zulia, con el propósito de programar la continuidad de la reducción del personal a través de la creación de microempresas.

En síntesis, la reducción del aparato estatal en las gobernaciones, da paso a una Administración Pública estadal inserta en un sistema red con empresas privadas, organizaciones del tercero y cuarto sector y microempresas, que cumplen funciones del Estado a través de contratos o convenios, se trata como dicen Sulbrant et al (2001: 119) de "un trabajo de unidades del sector público con unidades del sector privado o de organizaciones sin fines de lucro, estableciendo con ellas pautas de relaciones complejas, todas ellas importantes en la implementación de un número significativo de políticas públicas". Tales relaciones implican traslado de recursos materiales y capacidad de decisión del Estado a los otros miembros de la red, subordinación de las gobernaciones a los intereses de rentabilidad de las empresas privadas, y de las ONGs a las políticas estatales, alejando a estas organizaciones de los propósitos de defensa de los intereses colectivos para los cuales surgieron originalmente, convirtiéndose como sugiere Castells, en nuevas organizaciones gubernamentales. En general se trata de un entramado de relaciones aún no estudiadas y que escapan a los objetivos de este trabajo.

Según Castells (1998:12), el proceso de construcción del Estado red, lleva entre otros principios a la subsidariedad, según el cual, "la gestión administrativa debe situarse, para cada problema o tarea, en el ámbito más descentralizado posible en donde pueda desempeñarse eficazmente... 
En último término el principio de subsidariedad también lleva a sustituir el estado por la sociedad y la empresa en toda aquello en que el estado no sea necesario". A nuestro juicio el problema no es simplemente de eficacia o eficiencia, sino del uso de estos criterios sobre la base de reducción de costos y conflictos laborales y de tipo social.

Este proceso de reducción del aparato estatal se ha realizado, aplicando en el mejor de los casos, controles tradicionales de verificación de legalidad, sin los sistemas de control que algunas propuestas de la Nueva Gerencia Pública plantean como necesarias para garantizar condiciones de servicio efectivas, tales como el contrato o convenio de gestión, el cual según el CLAD (1998:16) "constituye un instrumento que permite, tanto una estimación más rigurosa de la eficiencia, de la eficacia y de la efectividad, como el aumento de la transparencia de la administración pública."

\section{Proliferación entes descentralizados administrativamente}

Otra línea de reforma del aparato de las gobernaciones a raíz del proceso de descentralización, es la creación de entes descentralizados administrativamente con mayor o menor grado de autonomía, para ejercer tradicionales y nuevas funciones de las gobernaciones.

La descentralización administrativa es una vieja estrategia organizativa de la administración pública nacional y municipal muy desprestigiada por diversas razones, entre ellas: altos costos, corrupción, clientelismo, elevado crecimiento burocrático, desvinculación de las políticas sectoriales, poder por encima del ente de quien depende jerárquicamente, en síntesis se han definido como organizaciones ineficaces, ineficientes e inefectivas. La búsqueda de soluciones a estos problemas giró siempre en torno a problemas de adscripción y de control, por ello las salidas siempre estuvieron en torno a la creación de una Ley de la Administración descentralizada, la cual nunca llegó a aprobarse, a pesar de los numerosos proyectos.

A pesar de esta situación, al lado de la desaparición de numerosos entes descentralizados vinculados al viejo modelo económico y al Estado de bienestar, así como producto de la privatización, encontramos el uso continuo esta estrategia organizativa, la cual es promovida por los proponentes de la Nueva Gerencia Pública. Según el CLAD (1998:15): "En las actividades exclusivas del Estado que permanezcan a cargo del Gobierno Central, la administración debe basarse en la desconcentración organizacional. Los organismos centrales deben delegar la ejecución de las funciones hacia las agencias descentralizadas. El principio que orienta 
este cambio es el de que debe existir separación funcional entre las estructuras responsables de la formulación de políticas y las unidades descentralizadas y autónomas, ejecutoras de los servicios, y de esta manera, orientar la administración pública a través del control de los resultados obtenidos por las agencias autónomas".

Las gobernaciones después de usar escasamente la figura de entes descentralizados, los utilizan para nuevas y viejas funciones, con distintas formas jurídicas: institutos autónomos, fundaciones, asociaciones civiles y una figura poco usada en el pasado como lo es, el servicio autónomo, el cual tiene autonomía bastante limitada en relación a las otras formas jurídicas.

La creación de entes descentralizados comienza a ser utilizada por las gobernaciones, para "gestionar" las competencias exclusivas recibidas del gobierno nacional a inicios de los noventa ${ }^{6}$, creando para ello institutos autónomos en la mayoría de las gobernaciones y servicios autónomos en el Estado Zulia y se van creando progresivamente fundaciones, asociaciones civiles e institutos autónomos para las competencias de tipo social y de promoción de actividades económicas. Desde los inicios del proceso de descentralización político-territorial las nuevas funciones de las gobernaciones se desarrollan a través de instituciones descentralizadas del aparato central, esto ha venido conformando estructuras organizativas cuyo rasgo común es la frondosidad de la administración descentralizada, incluso para áreas en las cuales tradicionalmente han existido Secretarías, tales como Salud, algunas Gobernaciones han constituido entes descentralizados, es el caso de la Gobernación de Carabobo.

Las propuestas de una Nueva Gestión Pública plantean la necesidad de controlar las agencias descentralizadas a través de Contratos de Gestión, el cual pasa por la definición de metas a priori para ser evaluadas, constituyendo la base de premios, sanciones y corrección de errores (CLAD, 1998: 16), sin embargo en este plano poco se ha avanzado. En la práctica los principales resultados de esta reforma organizativa han sido la flexibilización laboral en las instituciones vinculadas a la economía, en las cuales el número de trabajadores se ha reducido a su mínima expresión y el deterioro de las condiciones de trabajo, además de la flexibilización, en las Asociaciones Civiles y Fundaciones con funciones sociales ${ }^{7}$. La autonomía de estas organizaciones, les permite establecer relaciones flexibles con los trabajadores, difícil de lograr en el aparato central, esto ha venido atacando paulatinamente los criterios populistas y clientelares predominantes en la gestión pública. 
La reforma de los procesos administrativos ha tenido en las gobernaciones una dinámica distinta a la reforma dirigida a reducir el aparato estatal y a crear entes descentralizados las cuales han sido realizadas sin mayores discusiones. Por el contrario los intentos de modernización de procesos, lo que se ha llamado modernización administrativa, han sido lentos a pesar de la asesoría y financiamiento de organismos internacionales.

Desde 1990 el Gobierno Nacional a través de COORDIPLAN promovió la modernización de las Gobernaciones para ello se diseñó el Proyecto VEN/90/018 Gestión en Gobernaciones, Actualización y Dinamización (GAAD) con apoyo del PNUD, que centró la atención en la automatización de los sistemas y procedimientos, mecanismos de control de gestión, profesionalización del personal y mejoramiento de la estructura organizativa. A través de este proyecto el gobierno nacional desarrolló el interés de las gobernaciones en la modernización administrativa con la orientación de los multilaterales. De este proyecto participaron doce gobernaciones. Según Mascareño et al (2000:153), el papel que cumplió este proyecto fue de animador y difusor de la modernización de las gobernaciones. La estrategia utilizada fue la de realización de talleres con los directivos de las gobernaciones para la realización de diagnósticos en los cuales surgieron problemas en torno a las áreas de interés del proyecto GAAD, a la vez que se hicieron propuestas. Se logró por esta vía una motivar a las gobernaciones no sólo en el inicio de procesos de cambio administrativo, sino en perfilar una determinada orientación para el cambio.

Otros organismos nacionales promovieron la modernización administrativa de las Gobernaciones, el Programa de Inversión Social Local (PROINSOL), ejecutado por el Fondo de Inversión Social (FONVIS), también con presencia de los multilaterales, concretamente con financiamiento del BID y apoyo del PNUD, ejecutado desde 1993 se planteó como objetivo "apoyar y fomentar la descentralización de los servicios públicos, la racionalidad de la inversión pública en la infraestructura social local, el mejoramiento del gasto social y el fortalecimiento institucional y financiero de gobernaciones y municipalidades" (GEZ/PNUD-FONVIS, 1996:12). En el marco de este proyecto iniciaron un proceso de modernización, en 1993 las Gobernaciones de Falcón, Trujillo, Cojedes, Anzoátegui y Sucre y en 1997 las gobernaciones de Guárico, Nueva Esparta, Zulia, Barinas, Portuguesa y Mérida.

Si bien hubo diferencias entre una y otra gobernación debido a la incorporación de sus directivos en la elaboración del diagnóstico y de propuestas, en definitiva éstas giraron en torno a la automatización de los 
procesos, gestión de recursos humanos y reforma de la estructura organizativa, áreas de interés del PNUD. Pero realmente lo que definió las acciones de modernización con apoyo de los multilaterales, fue la automatización de los procesos y dotación de equipos, con impacto en el control de los trabajadores y en la atención de usuarios, tales como los proveedores. La prioridad a la automatización, se ha producido en algunos casos, conflicto con criterios de las gobernaciones, es el caso del Zulia, donde a pesar de no poder avanzar en un sistema de Dirección superior que constituyó el interés central de la gobernación, sobre cuya base se levantaría el modelo, se impuso la automatización. Según Obregón (2000: 25) "la automatización se inició mucho antes que se hubieran diseñado y reglamentado los procesos administrativos y estos se desarrollaron bajo las reglas de una misión institucional que la misma Gobernación cuestiona y que está en proceso de revisión", esto evidencia que existe en el fondo propósitos de homogenización de los procesos que se impone por la vía de la automatización, con la mediación del gobierno central y la participación de los multilaterales, lo que está resultando conflictivo en razón de las múltiples diferencias que existen entre las gobernaciones a raíz de la elección directa de los gobernadores y en consecuencia la posibilidad de influencia de diversas posiciones políticas e intereses en juego. La estrategia de apoyo de los organismos multilaterales hoy en día, incluye su participación hasta el proceso de implementación, lo cual ha garantizado que sus criterios sean puestos en práctica.

Por la vía de recursos propios y con otras fuentes de financiamiento externo, a las cuales acudieron individualmente las Gobernaciones, tales como el ILDIS y la Agencia Española de Cooperación, las Gobernaciones avanzaron en otros temas entre los cuales se destacan la creación de sistemas de rendición de cuentas por resultados y la formación del personal, áreas que también forman parte de las propuestas de cambio que en el medio científico se realizan para la gestión pública.

Los intentos de conformación de un sistema de rendición de cuentas por resultados han constituido una cuestión clave en el proceso de modernización de algunas gobernaciones, es el caso del Zulia y Carabobo, en la primera bajo la asesoría de Carlos Matus, a lo largo de la década de los noventa y la del dos mil, con una breve interrupción por cambio de gobierno, el centro de atención ha sido, como hemos dicho anteriormente, la reforma del sistema de dirección superior incorporándose incluso en los temas de financiamiento por los multilaterales. En dicho sistema se identificaron siete dispositivos ${ }^{8}$.

En materia de formación de personal, todas las gobernaciones han emprendido de una u otra forma procesos de capacitación de su personal con distintas fuentes de capacitación, pero paralelamente se realizan esfuerzos en cuanto a la creación de infraestructura sistemática en torno 
a esta materia. Son parte de este proceso de modernización impulsado por las gobernaciones sin la mediación de criterios nacionales, la creación del Instituto Zuliano de Estudios Políticos, Económicos y Sociales (IZEPES) como Escuela de Gobierno por parte de la Gobernación del Estado Zulia, el Centro de Desarrollo del Servidor Público del Estado Sucre desde donde se avanza en la formación de una nueva cultura administrativa.

\section{A modo de conclusión}

Asistimos desde comienzos de la década de los noventa a la realización de esfuerzos importantes en torno a la conformación de una nueva gestión pública en las gobernaciones, la cual lentamente va sustituyendo el paradigma burocrático populista y clientelar caracterizador de la administración pública venezolana a lo largo de cuatro décadas.

La nueva gestión pública tiende a la conformación de instituciones reducidas a su mínima expresión debido a la transferencia de funciones al sector privado y a la sociedad civil, insertándose así las gobernaciones en redes con organizaciones del sector privado y la sociedad civil, para cumplir las funciones que le son propias, en relaciones complejas de subordinación del Estado a intereses del sector privado y de subordinación de las ONGs a intereses del Estado y de particulares, proceso en el cual el Estado se va desprendiendo de sus responsabilidades de desarrollo y bienestar social, el cual es trasladado discretamente a la sociedad civil.

Se perfila además una nueva gestión pública con procesos automatizados, para ello ha recibido apoyo de varios organismos multilaterales, lo que se ha llamado modernización de los procesos administrativos es fundamentalmente automatización sobre la base de criterios, respecto a los procesos, impuestos por los organismos financieros, pasando en algunos casos, por encima de las propuestas de las gobernaciones.

Otro esfuerzo con cierto avance, gira en torno a la flexibilización laboral, facilitado con la proliferación de entes descentralizados administrativamente, con ambigüedades jurídicas, pero no es la única vía, se usan diversas estrategias dirigidas a la reducción de costos laborales así como de conflictos, tales como la conversión de los trabajadores en microempresarios, además de las formas predominantes hoy en día para flexibilizar las relaciones laborales.

La separación entre instancias de formulación de políticas e instancias ejecutoras es otra tendencia de la nueva gestión pública de las gobernaciones, el crecimiento organizativo necesario para asumir las nuevas funciones a raíz del proceso de descentralización político-territorial se ha dado fundamentalmente con la creación de entes descentralizados bajo diversas forma jurídicas. 
La rendición de cuentas por resultados ha ocupado la atención de los modernizadores en las gobernaciones, con avances lentos a pesar de la búsqueda de separación de los niveles de formulación de políticas y de implementación y del traslado de funciones al sector privado y a la sociedad civil, persistiendo sistemas de control tradicionales de verificación legal de los procesos.

En definitiva estamos en presencia de esfuerzos en torno a la reducción del aparato estatal, costos y conflictos laborales y sociales, con ausencia de incorporación de las estrategias de participación y control ciudadano, recomendadas fundamentales para avanzar hacia una gestión pública democrática, que promueva condiciones de justicia social.

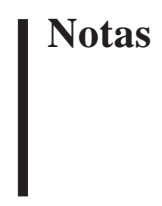

1 Estos son: Puerto Cabello, Maracaibo, La Guaira, Guanta, Guamache, Guaranao, Cumaná y Carúpano.

2 A modo de ejemplo encontramos que 265 empresas estaban contratadas en el año 2000 para prestar servicios en Puerto Cabello (IPAPC, 2000).

3 En el país existen un total de 497 aeropuertos entre públicos y privados, de los cuales 52 son del Estado, los más importantes son: Maiquetía (Caracas), la Chinita (Maracaibo) y Porlamar (Margarita), los cuales transportan el 90\% del total de pasajeros y carga internacional (www.cideiber.com). Pero no dejan de ser importantes los aeropuertos de Valencia y Barquisimeto por su condición de internacional.

4 Es útil señalar que con bastante frecuencia es un trabajo realizado por los trabajadores del sector privado, identificándose en su vestimenta con la institución del Estado.

5 En materia de Deporte encontramos que en el Estado Carabobo, "más de 100 organizaciones civiles se han incorporado a la ejecución de la política deportiva, especialmente la comunitaria" y en Desarrollo Social, en el Estado Lara "Más de 300 ONGs se han estructurado para servir de canales de intermediación, capacitación y defensa de las comunidades de base en las áreas sociales críticas como familia, nutrición, juventud" (Mascareño, et al, 2000: 129)

6 Se crearon entre otros los siguientes entes: En Carabobo: El Instituto Puerto Autónomo de Puerto Cabello, El Instituto de Aeropuertos del Estado Carabobo, el Instituto Autónomo Regional de Vialidad del Estado Carabobo (INVIAL), En el Zulia: El Servicio Autónomo de Recaudación y Manejo de los Ingresos del Puente General Rafael Urdaneta, Servicio autónomo Aeropuertos del Zulia, Servicio Autónomo Puerto de Maracaibo del Estado Zulia y Servicio Autónomo de Vialidad del Estado Zulia (SAVIEZ).

7 En un estudio realizado por Arguello (2000), a los entes descentralizados con funciones sociales en la Gobernación del Estado Zulia, se detectó que en estas organizaciones, la remuneración de un gran número de trabajadores no alcanzaba el salario mínimo, se encontraban en completa inestabilidad laboral, podían ser despedidos en cualquier momento sin ninguna explicación y los trabajadores creen laborar en ONGs por lo tanto no se consideran funcionarios del Estado. 
8 Los dispositivos fueron: La Agenda del Gobernante, el Procesamiento tecnopolítico, Manejo de Crisis, Planificación estratégica, Centros de Gran Estrategia, Monitoreo, Presupuesto por Programas, Petición y Rendición de Cuentas por resultados, Gerencia por Operaciones y Escuela de gobierno.

\section{Referencias bibliográficas}

Arellano Gault, David et al. Nueva gerencia pública en acción: procesos de modernización presupuestal. Un análisis inicial en términos organizativos (Nueva Zelandia, Reino Unido, Australia y México). In: Reforma y Democracia. 2000, n. 17, p. 7-43.

Arguello, Iris. Modernización Administrativa del Sector Social en la Gobernación del Estado Zulia. El Caso de los Entes Descentralizados. Tesis para optar al Título de Sociólogo en La Universidad del Zulia. Maracaibo. Venezuela 2000.

BARZELAY, Michael. La Reforma de la Administración Pública: Un Enfoque de políticas (con referencia a los países de la OCDE). Reforma y Democracia. 1998, n. 10, p. 113-128.

La Nueva Gerencia Pública. Un ensayo bibliográfico para estudiosos latinoamericanos (y otros). Reforma y Democracia 2001, n. 19, p. 7-66.

Boulton, Henry, et al. Informe Final TALLER IIC: TRANSPORTES, V1AS, FERROCARRILES, PUERTOS Y AEROPUERTOS. Foro Empresarial de las Américas. Cartagena. 1996. www.sice.oas.org . Consulta: 13-2-2002.

CAstells, Manuel. Hacia el Estado Red?. Globalización Económica e Instituciones Políticas en la era de la información. Ponencia presentada en el Seminario Internacional Sociedad y Reforma del Estado. Ministerio de Administración Federal y Reforma del Estado. Sao Paulo 26-28 de marzo de 1998.

CIDEIBER. Centro de Información y Documentación Empresarial sobre Iberoamérica. www.cideiber.com . Consulta: 04-03-2002.

CLAD. Una Nueva Gestión Pública para América Latina. CLAD. Caracas. 1998

Fuenmayor, Jennifer; Ochoa Henríquez, Haydée. Descentralización y Modernización Administrativa de las Gobernaciones en Venezuela. El caso del Estado Zulia. Ponencia presentada en el II Coloquio Internacional Políticas y Organizaciones Públicas en las Transformaciones del Estado a Nivel Regional. Universidad de Córdoba. 6 y 7 de diciembre de 2001 .

Guerrero, Omar. Del Estado Gerencial al Estado Cívico. Primera Edición. UNAM. México. 1999.

Kliksberg, Bernardo. Gerencia Pública en Tiempos de Incertidumbre. Instituto Nacional de Administración Pública. Madrid. 1989

Mascareño, Carlos, et al. Balance de la Descentralización en Venezuela: Logros, Limitaciones y Perspectivas. Documento del ILDIS. Caracas. 2000.

Obregón CAMpagna, Sonia Alicia. Fortalecimiento Institucional con asistencia financiera externa: El programa de Inversión Social Local (PROINSOL). MODERNIZACION. 2000, 
Obregón Campagna, Sonia; Carucci Tramonti, Flavio. El Proceso de Modernización de las gobernaciones Venezolanas: La Experiencia del Estado Zulia. Documento de la Fundación para la Modernización de las Administraciones Públicas de la Gobernación del Estado Zulia. Maracaibo. Venezuela. 2000.

OchoA Henríquez, Haydée; LóPez V alladARes, Mirtha; RodríGuez, Isabel. Administración Pública y Populismo en Venezuela. Revista Venezolana de Gerencia. Año 1, n. 1 p. 39-58.

Ochoa Henríquez, Haydée; Gamboa Cáceres, Teresa. Hacia la Gerencia Pública en Venezuela. El caso de la Administración Tributaria. Revista Centroamericana de Administración Pública. 1999, n. 37, p. 103-116.

Ochoa Henríquez, Haydée. Descentralización y retiro del Estado de la prestación de servicios en Venezuela. Ciencias de Gobierno. 2001, Año 5, n. 9, p. 99-121.

Ochoa Henríquez, Haydée; Rodríguez, Isabel. Descentralización y Privatización de la Salud en Venezuela. Estudio de un caso. En Tendencias. 2001, Vol. II , n. 2.

Petras, James. Duro alegato de James Petras contra las ONG's Principal. España/País Vasco.

Sulbrant, José; Lira, Ricardo; IbARra, Américo. Redes interorganizacionales en la administración pública. Reforma y Democracia. 2001, n. 21, p. 117-142. 
Revista do

Serviço

Público

Ano 54

Número 1

Jan-Mar 2003

Haydée Ochoa

Henríquez es

doctora en

Estudios del

Desarrollo,

investigadora

del Centro de

Estudios de la

Empresa de la

Facultad de

Ciencias

Económicas y

Sociales de la

Universidad del Zulia

(Venezuela) y directora de la Revista

Venezoelana de Gerencia.

Contato:

haydeeochoa@ iamnet.com
Abstract

\section{Governanças e Nova Gestão Pública na Venezuela \\ Haydée Ochoa Henríquez}

Ao final dos anos 80, o Estado venezuelano iniciou uma descentralização políticoterritorial, produzindo trasformações nos governos, instâncias receptoras das competências nesse processo. Os governos realizam reformas por meio de estrategia seletiva, incorporando algumas das propostas da nova gestão pública. Este trabalho explora essas reformas. Observam-se: diferenças entre os serviços sociais e os relacionados diretamente com a economia; privatização desses serviços; transferência à sociedade civil de alguns serviços sociais, criação de entes descentralizados; automação de processos; flexibilazação laboral; iniciativas com lento avanço na prestação de contas por resultados e capacitação para nova cultura administrativa. Em conclusão: a tendência é a conformação da gestão pública eficiente sobre a base da redução da burocracia estadual, de custos sociais, assim como de custos e conflitos laborais e escassa incorporação das propostas de participação que permitem consolidar a democracia e promover o bem-estar social.

\section{Gobernaciones y Nueva Gestión Pública en Venezuela Haydée Ochoa Henríquez}

A fines de los '80, el Estado venezolano inició una descentralización político-territorial produciendo cambios en las gobernaciones, instancias receptoras de competencias en este proceso, las cuales realizan reformas a través de la estrategia selectiva, incorporando algunas de la propuestas de la nueva gestión pública. Este trabajo explora estas reformas. Se observan: diferencias entre los servicios sociales y los relacionados directamente con la economía; privatización de éstos; traspaso a la sociedad civil de algunos servicios sociales, creación de entes descentralizados; automatización de procesos; flexibilización laboral; iniciativas con lento avance en rendición de cuentas por resultados y capacitación para una nueva cultura administrativa. En conclusión: la tendencia es a la conformación de una gestión pública eficiente sobre la base de la reducción del aparato estatal, de costos sociales, así como de costos y conflictos laborales y escasa incorporación de las propuestas de participación que permiten profundizar la democracia y promover el bienestar social.

\section{Governances and New Public Management in Venezuela \\ Haydée Ochoa Henríquez}

By the end of the 80's, the Venezuelan State initiated a territorial-political process of decentralization that led to changes in state governances. These state governances dependencies are the ones responsible for conducting reforms throughout selective strategy which incorporates some of the proposals made by the new public management. This article explores such reforms in terms of eight issues: differences between social services and those related to economy; privatization of such services; transfer of some social services to civil society; creation of decentralized agencies; automation of procedures; labor flexibility; initiatives with slow advance of reports based on results; and training in a new administrative culture. The article concludes that there is a trend to create an efficient public management on the basis of reduction of state bureaucracy, social costs as well as costs, labor conflicts and scarce participation of proposals leading to consolidate democracy and to promote social wellbeing. 